\title{
Research and Realization of Extracting the Attribute Values such as Boundary Points of Dimensions in AutoCAD Drawings
}

\author{
Jin $\mathrm{Su} *$, Yonghui Chen \\ Computer and Information Engineering College \\ Nanyang Institute of Technology \\ Henan Nanyang, China \\ sujinnylgedu@163.com \\ * Corresponding Author
}

\begin{abstract}
The method of extracting the dimension information of design drawing through AutoCAD ActiveX Automation technology is described. The additional information of cable bundle is saved and required in the way of banding extended data, and the cable length is calculated automatically using recursive algorithm. The core technology of the professional software research such as cable bundle design is solved, which can provide technical reference for the study of the similar projects.
\end{abstract}

Keywords- ActiveX Automation Fechnology; Dimension; Extended Data; Cable Bundle Design; Automobile Wiring Harness

\section{INTRODUCTION}

Wire harness design is mainly used in the development of automobile, ship, aircraft and other equipment control system. In the late stage of beam design, the design of the production process is usually to extract the jacket and line number information, and then calculate the length of each line, and the length of the line end, the use of pressure parts and waterproof plug and other process parameters, the final form of the main line of the production of the main line of the table, jacket, etc.

Automobile wiring harness is the main body of the circuit of the car, there is no wire harness and there is no car circuit [1]. At present, regardless of the advanced luxury car or economic type of ordinary car, the form of wire harness is basically the same, are from the wire, the plug and the composition of the tape. Electric wires are also known as low voltage wires, which are not the same as ordinary household electric wires. Ordinary household electric wires are copper single wire core, has certain hardness.

The whole working process is complicated, easy to make mistakes and omissions; especially in the calculation of a large number of pipelines length is time-consuming and laborious, seriously affecting the efficiency of product development, which takes up most of the process design personnel and energy [2]. Therefore, it can develop a set of software for the process design of wire harness, realizing the function of automatic calculation of pipeline length, automatic matching line fitting, etc. In this paper, we have made a deep research on the automation and accuracy of the production process design and process data generation of electrical wiring harness drawings, and put forward the solution of how to calculate the core technology of the pipeline length. At the same time, we give the core code, and provide technical support for the development of similar software.

\section{EXTRACT AUTOCAD INFORMATION}

Online beam in the design drawings size annotation mark of the drawings of the protecting is sleeve assembly relation and connection length. Therefore, the accurate calculation of the jacket inside, the length, primary condition is extracted drawings all labeling information to the database as the basis for the length calculation.

The AutoCAD interface of the ActiveX interface can not be directly obtained by the VBA programming, and the boundary point coordinates of the labeled object in the model space can be obtained directly [3]. By analyzing the realization principle of AutoCAD, the labeling objects in the model space are corresponding to the block space. Through the experiment, it is found that the basic graphic elements can be obtained by the program, and the coordinate of the object can be extracted accurately, as is shown by equation (1).

$$
\begin{aligned}
X(m+1) & :=\left[x^{T}(m+1,1), x^{T}(m+1,2), \cdots, x^{T}(m+1, M)\right]^{T} \\
& :=\left[x^{T}(m M+1), x^{T}\left((m M+2), \cdots, x^{T}(m M+M)\right]^{T}\right.
\end{aligned}
$$

But in the block space, the space of the label and the model space correspond to the different object handles, so it is also a technical problem that how to extract the label information and the corresponding model space [4]. It is found by experiments that the insertion point coordinates can be extracted from the model space, and the insertion point coordinates of the annotated text can be obtained within the block space, and there is no more than one. Therefore, it can be solved by using the decision block space and model space.

\section{EXTENDED DATA ACCESS GRAPHICS OBJECT ADDITIONAL INFORMATION}

Active $\mathrm{X}$ technology can be used in any graphics element to bind some additional properties, such as 
material encoding, description, color, line, and other information.

AutoCAD within the drawing of the sheath and line number are ordinary text objects, can not express the details of the electrical properties of the components. And through the two development of AutoCAD, the additional attribute information of the electrical components can be attached to the corresponding drawing object. In the future, it can not only extract the electrical components information, but also can extract the corresponding technical parameters of the electrical components, and provide the basic data for the later process design, as is shown by equation (2) [5].

$$
\{\mathbf{z}(k)\}=\left\{\left[\begin{array}{c}
\mathbf{x}_{1}(k) \\
\vdots \\
\mathbf{x}_{n}(k)
\end{array}\right]\right\}
$$

AutoCAD graphics objects are provided with GetXData and SetXData method, which can achieve the additional information to the corresponding graphics objects, binding data to the graphics object program main code, is as follows:

Dim DataType (0 To 3) As Integer //Declare extended data type variable

Dim Data (0 To 3) As Variant // Declare extended data variable

DataType $(0)=1001$ : Data $(0)=$ "LineNoInfo" // Line number correlation information

DataType $(1)=1071$ : Data $(1)=$ LineHandle $/ / 32$ bit integer, line number, handle

DataType (2) = 1000: Data (2) = LineColor // Character type, line color

DataType (3) = 1040: Data (3) = LineRadius // Float type, line diameter

elem. SetXData DataType, Data

The main program code for the acquisition of the graphics object binding additional data is as follows:

Dim DataType As Variant // Declare extended data type variable, must be a variant type

Dim Data As Variant // Declare extended data variable

txtX.GetXData "LineNoInfo", DataType, Data

If Not IsEmpty (Data) Then // If you have specified binding data

LineNoID = CLng ("\&H" \& elem.Handle) //

Convert current object handle

If LineNoID = Data (1) Then // If the object handle is in line with the binding handle

LineColor= Data (2) // Line color

LineRadius= Data (3) // Wire diameter End if

$$
\text { End if }
$$

\section{AUTOMATIC CALCULATION OF PIPELINE LENGTH BY RECURSIVE ALGORITHM}

Because of the drawings in the pipeline are included in the concrete sheath, the casing is connected to the corresponding tagging boundary point [6]. Therefore, the calculation of the actual length of any pipeline, pipelines at both ends of the sheath corresponding to the annotation of boundary points respectively as the start point and end point, according to the already provided from database annotation information database, using "fiddling" retrieval methods one by one analysis of the current connection at both ends of the pipeline connection point of the annotation information, and realizes the automatic calculation of the length of the pipeline to the recursive algorithm and program control processes such as shown in Figure 1.

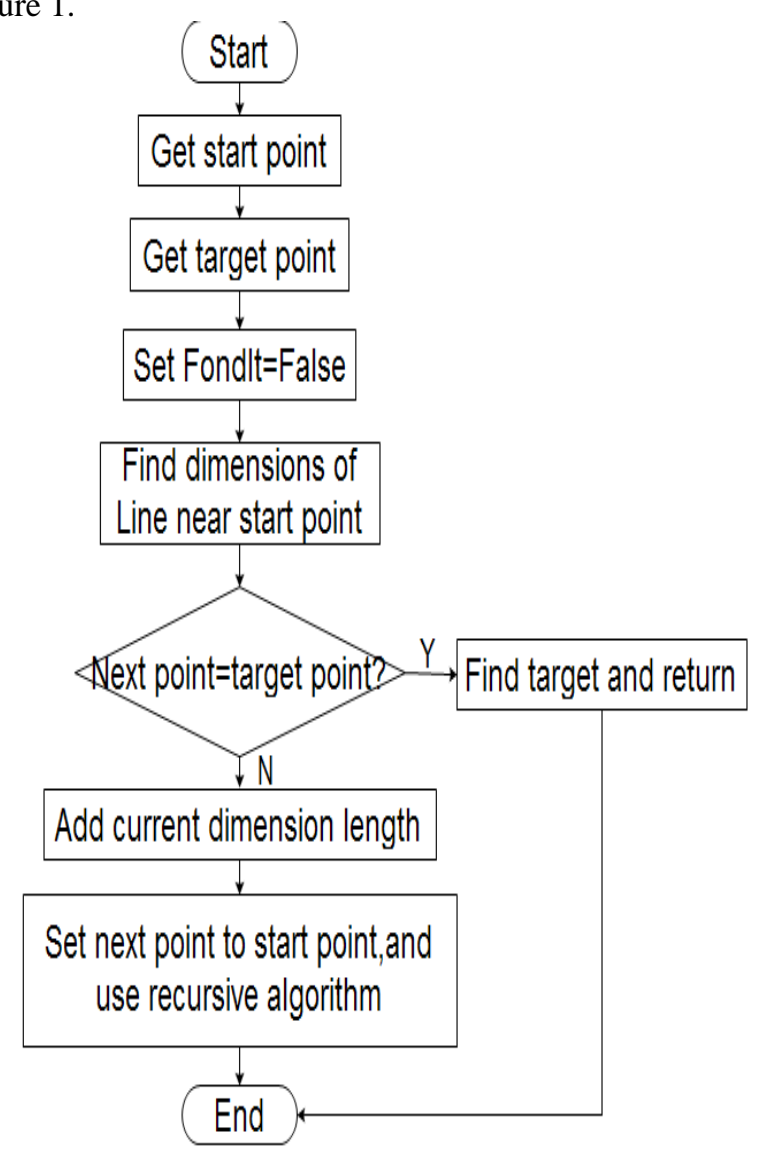

Figure 1. Calculation of Pipeline Length Program Flow Chart.

Calculate the function function of the assembly length between two points:

Private Function GetLength (StartPX as Long, StartPY As Long, DimHandleID As Long) As Long

Dim rr As New ADODB.Recordset

If FondAim Then GetLength = 0: Exit Function

First, the boundary points in the table are retrieved.

StartPX, Start PY Label information

$\mathrm{sql}=$ "Select $*$ from table Where $((\mathrm{P} 1 \mathrm{X}="$ \& StartPX \& " and P1Y=" \& StartPY \& ") or (P2X=" \& StartPX \& " and $\mathrm{P} 2 \mathrm{Y}="$ \& StartPY \& ")) and HandleID $<>"$ \& DimHandleID

rs. Open sql, Conn, adOpenForwardOnly, adLockReadOnly

If rs.EOF Then Exit Function

While not rs.EOF

If FondAim Then Exit Function

GetLength = rs ("Length")

If StartPX = rs ("P1X") And StartPY = rs ("P1Y") Then

(1) Then 
FondAim = True: Exit Function // If you have found the target coordinates, exit

End If // If you have not found the target coordinates, the recursive call to the calculated length function

GetLength = GetLength + GetLength (rs ("P2X"), rs ("P2Y"), rs ("HandleID"))

Else

If rs $(" \mathrm{P} 1 \mathrm{X} ")=$ EndPnt (0) And rs("P1Y") = EndPnt (1) Then

FondAim = True: Exit Function // If you have found the target coordinates, exit

End If

GetLength = GetLength + GetLength (rs ("P1X"), rs ("P1Y"), rs ("HandleID"))

End If

rs.MoveNext

Wend

End Function

The wiring harness diagram is a full vehicle circuit diagram drawn according to the actual installation site of the electrical equipment in the car [7]. In the drawing, the wire between the components and the components is in the form of wire harness, as is shown by equation (3).

$$
\left.\begin{array}{rl}
Z_{1}= & H_{1} X+V_{1} \\
Z_{2}= & H_{2} X+V_{2} \\
& \cdots \cdots \\
Z_{r}= & H_{r} X+V_{r}
\end{array}\right\}
$$

The wire drawing and the drawing line are similar, but the picture is simple and clears [8]. Harness installation diagram is described in detail to the internal harness wire, only the DEW line beam outside the thread and plug device with numbers, and used the letters "calibration [9].

In the main program call GetLength () function code is as follows:

Dim LineNoLenth as Long

Dim FondAim as Boolean // Whether to find the target point

Dim StartPnt (0 To 2) As Long // Calculate the length of the starting point coordinates (exact to integer)

Dim EndPnt (0 To 2) As Long // Calculate the length of the end point coordinates (exact to integer)

StartPnt (0)=100:StartPnt(1)=100 // Assignment to calculate the starting point of the length of the pipeline

EndPnt $(0)=1200:$ EndPnt(1)=4600 // Assignment to calculate the length of the pipeline end coordinates

FondAim=False // Set up the identification variable

LineNoLenth $=$ GetLength $(\operatorname{StartP}(0), \operatorname{StartP}(1), 0)$

if FondAim then

MsgBox " Calculate the success, the current length of the pipeline is:" \& LineNoLenth, vbOKOnly, "Calculation length"

Else

MsgBox "The calculation fails, the current line at both ends of the pipeline can not be connected.", vbOKOnly, " Calculation length"

End if
Automotive wiring harness design basic idea is that according to the use of electrical equipment, in particular the high power equipment including each sensor signal voltage current, first determine the maximum line current, according to the current determine the wire harness section, determine the length of the wire harness, and then calculate the total current to determine the main fuse current, select the fuse, as is shown by equation (4) [10].

$$
R\left(W_{j}^{\sigma}, F_{e}\right)=\frac{E\left[\left(\xi_{W_{j}^{\sigma}}-E\left(\xi_{W_{j}^{\sigma}}\right)\right)\left(\xi_{F_{e}}-E\left(\xi_{F_{e}}\right)\right)^{T}\right]}{\sqrt{D\left(\xi_{W_{j}^{\sigma}}\right) D\left(\xi_{F_{e}}\right)}}
$$

In the selection of good after the use of threedimensional software design wire harness, and it is according to the three-dimensional wire harness production line.

Calculate the length of the pipeline function interface as shown in figure 2 .

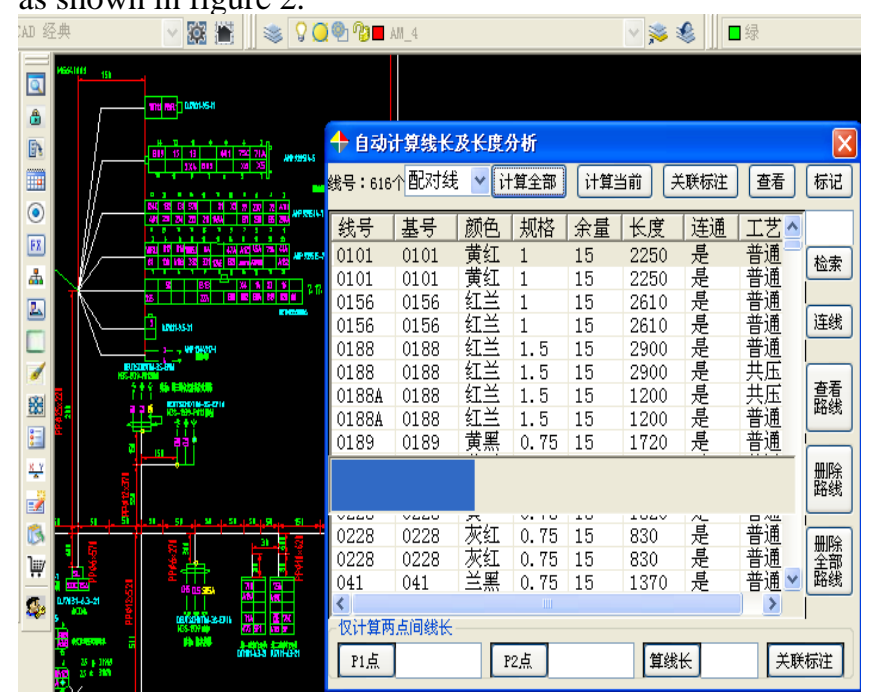

Figure 2. Calculate the Length of the Line

\section{SUMMARY}

Automatic calculation of the actual length of the pipeline between the engineering drawings is one of the main functions of the wire harness design software, the current domestic such software is rarely. And accurate access to the drawing information and the calculation of the length of the graphics object is the key technology of this kind of software development.

In this paper, we study the corresponding relationship between the model space and the space of the AutoCAD, and propose the important information from the block space to obtain the label boundary points. Then, according to the label of the text, we can find the correct information of the object. By analyzing the characteristics of the connection between the lengths of the graphic object, a recursive algorithm is used to calculate the length of the connection between any two lines. Currently the technology has been successfully applied to Zhengzhou Yutong Bus company developed harness aided design software, the software in the test and practical application of the enterprise, stable operation of the program calculated pipeline length of speed and high precision. 


\section{REFERENCES}

[1] Wirawan Sumbodo, Boenasir, Karsono, Agung Pambudi, "The Making of Workpieces Using Autocad Software based Siemens Sinumerik 802C Base Line Frais Machine", IJEI, Vol. 2, No. 2, pp. $35 \sim 42,2011$

[2] Zlatan Stojkovic.Application of Software Tools in Power Engineering Calculations. Computer-Aided Design in Power Engineering,2012.

[3] Rain Chen, "Using Multi-Dimensional Scale to Display Design Patent Layout", AISS, Vol. 5, No. 11, pp. 283 292, 2013.

[4] Chen Li. Research on Distributed Synchronous CAD Collaborative Design System. Frontier and Future Development of Information Technology in Medicine and Education,2013.

[5] Hujun He. Establishment of Dynamic Visualization Legend Library in the AutoCAD Environment Based on VBA. Proceedings of the International Conference on Information Engineering and Applications, 2013.
[6] Hong-Kyu Kwon, Kwang-Kyu Seo, "CAE simulation of HPDC Process with Automobile part (Oil Pan)", JDCTA, Vol. 7, No. 13, pp. $245 \sim 251,2013$.

[7] Marian Mešina. Visualisation of Semantic Networks and Ontologies Using AutoCAD. Cooperative Design, Visualization, and Engineering,2014.

[8] Li Shunxi, Wang Aimin, "An Evaluation of Chinese Automobile Manufacturers Using the Analytic Hierarchy Process and Catastrophe Theory", AISS, Vol. 4, No. 1, pp. 28 36, 2012.

[9] M. J. Sánchez-Granero. Introducing fractal dimension algorithms to calculate the Hurst exponent of financial time series. The European Physical Journal B,2012.

[10] L. V. Bulanov. Engineering method of calculating an efficient length for the vertical section of a continuous caster on which the semifinished product is bent and straightened. Metallurgist,2006. 